\title{
Actualidades farmacológicas en el tratamiento de la insuficiencia cardiaca crónica
}

\author{
Juan Antonio Ornelas Garnica*
}

\begin{abstract}
RESUMEN
La insuficiencia cardiaca es, actualmente, una de las grandes epidemias del nuevo siglo. Durante las últimas dos décadas se ha incrementado de manera dramática y exponencial, ubicándose como la primera causa de morbimortalidad y hospitalización a nivel mundial hasta en un 159\% y ocupando $10 \%$ de morbilidad en la población mexicana. Es por esto que en la actualidad se ha modificado drásticamente el rumbo del tratamiento farmacológico, dejando de lado la paliación de las complicaciones propias de la evolución del padecimiento, enfocándose hacia la identificación y ruptura de los eslabones más precisos que conforman la cadena de acontecimientos adaptativos fisiológicos desencadenados por la misma, los cuales, durante los últimos años, se ha demostrado que son causantes directos de la progresión patológica de la enfermedad y la exacerbación de un sistema de retroalimentación positivo que se magnifica a sí mismo. En la presente revisión se contemplan los diferentes mecanismos compensatorios de la insuficiencia cardiaca, como los objetivos directos del tratamiento farmacológico actual, partiendo de los archivos de la Revista Española de Cardiología y la Revista Mexicana de Medicina Interna. Con este nuevo enfoque se pretende aminorar la mortalidad y los índices de hospitalización de manera importante, y al mismo tiempo mejorar la calidad de vida de las personas que la padecen.
\end{abstract}

Palabras clave: Insuficiencia cardiaca, epidemia, mecanismos compensatorios, morbimortalidad.

\section{News in the pharmacological treatment of chronic heart failure}

\begin{abstract}
Heart failure is currently one of the great epidemics of the century, that during the past two decades has increased dramatically and exponentially, ranking as the leading cause of morbidity and hospitalization worldwide up to $159 \%$ and ranking $10 \%$ morbidity of the Mexican population. That's why today has changed dramatically the course of drug treatment, palliation aside from the complications of the disease evolution, toward identifying and breaking most accurate links in the chain of events adaptative physiological triggered by it, which in recent years has proven to be the direct cause of pathological progression of the disease and the exacerbation of a positive feedback system is magnified himself. Thus, in this review referred to the various compensatory mechanisms of heart failure, such as direct targets of current drug treatment, starting from the archives of the Spanish Journal of Cardiology and internal Medicine Mexican Magazine. With this new approach seeks to reduce mortality and hospitalization rates significantly, while improving the quality of life of people who have it.
\end{abstract}

Key words: Heart failure, epidemic, compensatory mechanisms, morbidity and mortality.

*Lic. Enf. Juan Antonio Ornelas Garnica.

Correspondencia:

E-mail: eneo_stardust@hotmail.com

Este artículo puede ser consultado en versión completa en http://www.medigraphic.com/enfermerianeurologica 


\section{INTRODUCCIÓN}

$\mathrm{L}$ a insuficiencia cardiaca (IC) es una entidad clínica compleja de padecimientos cardiovasculares; se manifiesta cuando el corazón no es capaz de mantener el bombeo adecuado para satisfacer las necesidades metabólicas de la economía en cada momento, resultante de trastornos cardiacos estructurales o funcionales que deterioran la capacidad del ventrículo para llenarse o para expulsar la sangre. ${ }^{1}$

En algunas ocasiones, la IC se desarrolla súbitamente y puede afectar el lado derecho, el izquierdo o ambos del corazón. Al perderse la acción de irrigación al corazón, la sangre se dirige a otras partes del cuerpo como el hígado, el tracto gastrointestinal, las extremidades y los pulmones. $1^{\circ}$. Implica falla o defecto en la contractilidad del músculo cardiaco, $2^{\circ}$. Es la incapacidad del sistema cardiovascular de realizar funciones básicas como irrigar los tejidos para llevar los nutrientes necesarios y a su vez eliminar los productos de desecho.

Así mismo, la congestión circulatoria secundaria a la retención hidrosalina anormal, sin alteración intrínseca de la función cardiaca, se denomina "estado congestivo". Generalmente, la IC es consecuencia de una insuficiencia miocárdica, y a su vez insuficiencia circulatoria, aunque en ocasiones se presentan cuadros de hipovolemia severa, en la que existe falla circulatoria, sin falla cardiaca, por lo que pueden existir manifestaciones clínicas similares, pero en su origen y fisiopatología son entidades distintas y, por tanto, el tratamiento puede variar en cada una de ellas.

El estudio más reconocido sobre la prevalencia es el de Framingham, quien señaló para el grupo de edad de 50 y 59 años una prevalencia de $0.8 \%$, que se incrementa a $2.3 \%$ para personas de entre 60 y 69 años, $5 \%$ para las de 70 a 79 años y $10 \%$ para las de 80 años y más. ${ }^{2}$ Abdhelafis planteó que desde los 65 hasta los 75 años de edad, más de $5 \%$ de las personas la padecen, y para mayores de 80 años la cifra estaría entre 10 y $20 \%$. En España, más de $80 \%$ de los casos acontecen en mayores de 65 años; su prevalencia alcanza $10 \%$ en los mayores de 70 años y es la primera causa de hospitalización en este grupo de edad. ${ }^{3,4}$ Además de estas estimaciones, existen otras basadas en la incidencia de razas ubicando a negros y mestizos en $46 \%$, blancos $52 \%$ y otros en $0.9 \%$. Aun cuando estas cifras no son definitivas, al menos permiten entablar las relaciones de incidencia en otra perspectiva.

Actualmente existen dos tipos fundamentales de insuficiencia cardiaca crónica (ICC) atendiendo a la función del ventrículo que esté alterada. La insuficiencia cardiaca sistólica (ICS) manifiesta un déficit de función contráctil del miocardio, dejando al margen a la insuficiencia cardiaca diastólica (ICD); aquí el problema es un déficit de relajación ventricular. Es importante señalar que, según las tendencias actuales, para conseguir un efectivo diagnóstico, se basan en la determinación de la función sistólica del ventrículo izquierdo (FSVI); es por ello que ante la sospecha de lesión miocárdica, aun cuando el paciente se encuentre asintomático es fundamental explorar la FSVI para determinar el posible origen y tratamiento eficiente según su etiología, así como su pronóstico, mismos que se apoyan directamente en la ecocardiografía Doppler. En cuanto a la evolución natural de la insuficiencia cardiaca crónica sistólica (ICCS), ésta dirige la progresión de la disfunción ventricular izquierda. Los mecanismos de compensación del organismo ante la propia insuficiencia cardiaca (activación del eje renina-angiotensina-aldosterona, activación del sistema nervioso simpático, remodelado ventricular principalmente), si bien en un primer momento ayudan al organismo a superar el deterioro hemodinámico causado por el déficit de función del corazón, a largo plazo ejercen un importante efecto deletéreo de instauración paulatina y prácticamente irreversible.

\section{VALORACIÓN DE ENFERMERÍA DE LA ETIOLOGÍA}

Existen dos principales tipos de IC y se dividen en tres grupos: diastólica, sistólica y mixta. La primera se asocia a valvulopatías estenóticas, hipertensión arterial crónica y cardiomiopatía hipertrófica primaria; la segunda a miocardiopatía dilatada y cardiopatía isquémica, y la tercera a miocardiopatía restrictiva, principalmente. Las diferencias clínicas entre las dos primeras consisten en lo siguiente: Disfunción sistólica: insuficiencia contráctil con FSVI < $40 \%$, presencia de tercer ruido, dilatación cardiaca y tiene un predominio del $60 \%$. Disfunción diastólica: alteración en la relajación o repleción (afectando la precarga), FSVI normal, índice cardiotorácico normal, presencia de cuarto ruido e incidencia de $30-40 \%$.

En la actualidad, continúan vigentes los criterios diagnósticos del Grupo de Trabajo de la Sociedad Europea de Cardiología para la Insuficiencia Cardiaca Diastólica: 1) Síntomas y signos de insuficiencia cardiaca. 2) Función sistólica normal del VI normal (fracción de eyección mayor a 50\%). 3) Anomalías en la relajación, el llenado o rigidez diastólica del VI. ${ }^{5}$

\section{¿QUÉ SUCEDE DURANTE LA ENFERMEDAD?}

Con los avances de la medicina, se ha descubierto que cuando la función de bombeo del corazón se haya deprimida, se pone en marcha una serie de mecanismos compensadores para tratar de mantener el gasto cardiaco y asegurar el riego de los órganos jerárquicamente superiores, como la circulación coronaria y cerebral. Se presentan dos sucesos 
importantes que intervienen en la fisiopatología de la IC: la restructuración ventricular y la activación de varios sistemas neurohormonales provocando la progresión, los que a su vez son causantes de mortalidad a largo plazo. La sobrecarga de trabajo por exceso de tensión o de carga volumétrica y la pérdida de miocitos por daño isquémico (infarto) o miocardiopatía según la causa de lesión, inducen el proceso de restructuración que altera el tamaño, la forma y la función ventricular. En esto consiste la principal respuesta sistémica con el objetivo de restaurar la función ventricular.

Se reconocen tres formas de restructuración:

a) En respuesta a la sobrecarga de tensión, los miocitos se ensanchan y producen hipertrofia ventricular concéntrica; b) En la sobrecarga volumétrica ocurre el alargamiento de los miocitos con hipertrofia excéntrica, y c) La restructuración que ocurre después de un infarto miocárdico, que pudiéramos llamar "mixto", en el que se alternan zonas hipertróficas con zonas dilatadas. En este proceso de restructuración también intervienen, en cierta medida, la fibrosis por activación de fibroblastos y la apoptosis o muerte celular programada. ${ }^{6}$

\section{INTERVENCIÓN DE ENFERMERÍA PARA EL TRATAMIENTO}

La intervención del profesional de enfermería al proporcionar el tratamiento debe ser oportuna y tener presente que para ello se interponen varios estímulos en la aparición de la restructuración; éstos pueden ser: locales, de estiramiento de los miocitos y otros neurohormonales; aquí la intervención es proporcionar los medicamentos indicados oportunamente, como la angiotensina II, la noradrenalina, la aldosterona, la endotelina, el óxido nítrico, el factor de necrosis tumoral $\alpha$ (TNF- $\alpha$ ) y el factor de crecimiento tisular $\beta$ (TGF- $\beta$ ). ${ }^{7}$ De esta forma se producen alteraciones como: Activación Neurohormonal, Péptidos Natriuréticos, Sintetasa de Óxido Nítrico (NO), Citocinas proinflamatorias en IC, Metaloproteinasas de la matriz Extracelular y en la Endotelina.

\section{ACTIVACIÓN NEUROHORMONAL}

La activación crónica del sistema nervioso simpático del sistema renina-angiotensina-aldosterona y de la arginina-vasopresina es responsable de la mayoría de las anormalidades fisiológicas y clínicas; la IC se caracteriza por vasoconstricción excesiva, retención de sal y fluidos, incremento en la postcarga, arritmias, remodelación del ventrículo y deterioro progresivo de la función miocárdica. Paralelamente, los pacientes con IC demuestran una activación de las respuestas hormonales contrarregulatorias benéficas, como los péptidos natriuréticos y la adrenomedulina, que tienen propiedades inotrópicas, vasodilatadoras y natriuréticas. ${ }^{8,9}$ La aldosterona promueve la activación del sistema simpático, la inhibición del parasimpático y la fibrosis miocárdica y vascular.

Desde el punto de vista teórico, para revertir este daño inicial podrían utilizarse los siguientes fármacos: inhibidores de las enzimas convertidoras de angiotensina (IECA), bloqueadores adrenérgicos, antagonistas de la aldosterona, bloqueadores de los receptores de la endotelina, agentes antioxidantes y antagonistas del TNF- $\alpha$. Sin embargo, el tratamiento con antagonistas de la endotelina no produce beneficio clínico y puede relacionarse con la aparición de efectos adversos, sobre todo la hepatotoxicidad. ${ }^{10,11}$ Los antagonistas del TNF- $\alpha$ se consideran actualmente contraindicados, pues aumentan el riesgo de muerte $\mathrm{u}$ hospitalización en pacientes con insuficiencia cardiaca. ${ }^{12,13}$ Durante el proceso de evolución de la IC hay una activación del sistema simpático y neurohormonal, con liberación de sustancias como el péptido natriurético auricular, endotelina, factor alfa de necrosis tumoral, factor tisular, entre otros.

\section{PÉPTIDOS NATRIURÉTICOS}

Las hormonas natriuréticas (A, B y C) son una familia de péptidos vasoactivos; se sintetizan en el corazón ante situaciones de sobrecarga, provocando la distensión de los miocitos auriculares. Actúan en el riñón induciendo natriuresis para disminuir el volumen circulante $\mathrm{y}$, por retroalimentación, disminuir la distensión auricular. ${ }^{14}$

Una revisión sistemática de 36 artículos señala que el péptido natriurético tipo B (BNP) es un indicador pronóstico significativo para los pacientes con IC en cualquier estadio de la enfermedad; es el mejor indicador de supervivencia que muchos indicadores tradicionales tienen como clase funcional de la NYHA; la creatinina sérica y la fracción de eyección del VI. Ésta presenta riesgo relativo de muerte, que se incrementa en $35 \%$ por cada $100 \mathrm{pg} / \mathrm{mL}$ de elevación del BNP en estos pacientes. ${ }^{15}$

\section{SINTETASA DE ÓXIDO NÍTRICO (NO)}

La expresión y actividad de la sintetasa de NO está incrementada en la IC. La producción excesiva de NO acelera la degradación de AMPc e inhibe la entrada de calcio a través de los canales lentos de calcio, lo que se resume en un efecto inotrópico negativo. Los valores fisiológicos de NO parecen tener efectos positivos e inhiben ciertos mecanismos apoptóticos, mientras que las concentraciones patológicas ejercen efectos citotóxicos asociados a efectos inotrópicos y lusotrópicos negativos. 


\section{CITOCINAS PROINFLAMATORIAS EN IC}

Los pacientes que presentan incremento en citocinas y quimiocinas desempeñan un papel importante en el remodelamiento ventricular y en la progresión de la IC. Aunque las citocinas antiinflamatorias están incrementadas, ${ }^{16-18}$ la excesiva activación ejerce efectos deletéreos por los siguientes mecanismos:

a) Acción inotrópica negativa mediante la inhibición de la SERCA2a y la estimulación de iNOS.

b) Favorece el remodelamiento ventricular mediante la inducción de metaloproteinasas y de respuestas apoptósicas. ${ }^{19,20}$

Actualmente se cree que en el corazón, los niveles elevados de $\mathrm{TNF} \alpha$ pueden llegar a causar falla cardiaca letal asociada a miocardiopatía dilatada. Se ha encontrado una relación entre la evolución de la IC y los niveles de dicho factor; por otro lado, se especula si el TNF $\alpha$ puede desencadenar los resultados en la fase final y a la vez predecir el tipo de atención que requieren los pacientes, ya sea utilizando algún dispositivo de asistencia ventricular izquierda o la atención médica farmacológica avanzada, todo esto mientras el paciente se encuentra en la etapa de espera para un trasplante cardiaco.

\section{METALOPROTEINASAS DE LA MATRIZ EXTRACELULAR}

Existe una íntima relación entre la matriz de colágeno y los miocitos cardiacos. El colágeno miocárdico asegura la integridad de los miocitos aledaños. Los cambios adversos en la matriz contribuyen a la disfunción de la contractilidad y al remodelamiento ventricular en la IC. Se ha demostrado un incremento en la activación de las metaloproteinasas de la matriz (MPM) y un descenso en los inhibidores tisulares de MPM. ${ }^{21,22}$ El proceso de restructuración en los pacientes tiene dos fases. Primera: Los estímulos mecánicos de distensión parietal y la estimulación de los receptores neurohormonales de los miocitos inducen la hipertrofia adecuada a la situación; si ésta se mantiene crónicamente, la reserva funcional cardiaca se agota, los miocitos exhaustos comienzan a morir y la hipertrofia ya no es suficiente para compensar las demandas. Segunda: Se presenta dilatación ventricular. ${ }^{23}$

Con el agotamiento de la reserva cardiaca es inevitable la disfunción del ventrículo izquierdo, disminuyendo el gasto cardiaco y la tensión arterial sistémica. Esto a su vez activa diferentes sistemas neurohormonales compensadores para tratar de aumentar la tensión arterial y la perfusión tisular: el sistema barorreceptor-sistema nervioso simpático, el sistema reninaangiotensina-aldosterona y el sistema arginina-vasopresina.
Estos sistemas incrementan la frecuencia cardiaca y la contractilidad miocárdica, producen vasoconstricción periférica y provocan la retención de sal y agua. Inicialmente, estos mecanismos compensadores son capaces de mantener el gasto cardiaco y la tensión arterial, pero al paso del tiempo incrementan el trabajo miocárdico y la pérdida de miocitos, lo que origina un círculo vicioso que perpetúa los mecanismos y provoca la evolución de la enfermedad. Es por ello que las nuevas tendencias farmacológicas se basan en el conocimiento de estos mecanismos neurohormonales con el objetivo de bloquearlos e interrumpir el proceso de la enfermedad y con ello mejorar el pronóstico del paciente.

\section{ENDOTELINA}

Funciona de un modo inhibitorio, manteniendo la relajación del tono vascular e inhibiendo el crecimiento del músculo liso y la adhesión y agregación de leucocitos y plaquetas. La disfunción endotelial manifiesta un deterioro de la vasodilatación dependiente del endotelio; se ha demostrado en una variedad de alteraciones cardiovasculares, como hipercolesterolemia, hipertensión, insuficiencia cardiaca, aterosclerosis y diabetes. ${ }^{24,25}$

La disfunción endotelial en la IC puede ser atribuida a un descenso en la síntesis endotelial de ON y/o a un incremento en su degradación por radicales libres. La sobrecarga de presión y volumen, el incremento en citocinas inflamatorias y la activación neurohormonal también desempeñan un papel determinante en la disfunción endotelial en la IC. ${ }^{26,27}$

\section{CRITERIOS CLIINICOS A CONSIDERAR}

Los síntomas y signos de mayor relevancia clínica son: disnea de esfuerzo, ortopnea, disnea paroxística nocturna, reducción de la tolerancia al ejercicio, letargia, fatiga, tos nocturna, hinchazón de las piernas, anorexia, taquicardia, pulso alternante, distensión venosa yugular, latido de la punta desplazado, crepitancia o sibilancia, tercer o cuarto ruidos cardiacos, edema periférico, hepatomegalia sensible y/o ascitis.

Las nuevas definiciones de este síndrome se basan en los siguientes criterios:

1) Síntomas y signos de congestión o mala perfusión tisular, 2) Prueba objetiva de disfunción cardiaca mediante ecocardiografía u otras pruebas, 3) Respuesta al tratamiento dirigido a la disfunción. ${ }^{28,29}$

El American College of Cardiology y la American Heart Association emitieron una nueva clasificación de la insuficiencia cardiaca basada en el desarrollo y la progresión de la enfermedad: ${ }^{30,31}$ 
Cuadro I. Clasificación por estadios (ACC/AHA).

A: Alto riesgo de padecer insuficiencia cardiaca sin alteraciones estructurales aparentes del corazón

B: Anomalías estructurales sin síntomas de insuficiencia cardiaca

C: Anomalías estructurales con síntomas previos o actuales de insuficiencia cardiaca

D: Síntomas de insuficiencia cardiaca rebeldes al tratamiento estándar

\section{HALLAZGOS FíSICOS}

Los signos y síntomas dependerán del tipo de falla cardiaca y del tiempo de evolución de la misma; la falla cardiaca en el lado derecho del corazón (hipertensión venosa generalizada) se manifiesta por: a) Hepatomegalia o esplenomegalia, b) Edema periférico progresivo que puede llegar hasta ascitis o anasarca, c) Ingurgitación venosa yugular y d) A veces cianosis periférica; falla cardiaca lado izquierdo (hipotensión venosa pulmonar): Manifiesta a) Disnea progresiva hasta ortopnea, b) Disnea paroxística nocturna y c) Edema pulmonar. Cabe señalar que la falla cardiaca suele ser global, con mezcla de los dos tipos de falla; aunque en un inicio pudiera ser específica, en un momento dado puede afectar el sistema contiguo por diversos mecanismos. Entonces, los términos de "falla derecha" y "falla izquierda" se refieren teóricamente a la parte del corazón afectada en el comienzo de la enfermedad. Así mismo, la falla derecha a la larga se complica con disnea, derrame pleural y demás manifestaciones clínicas de la falla izquierda.

\section{LABORATORIO Y GABINETE}

Los exámenes de laboratorio ayudan al diagnóstico etiológico de la falla cardiaca; el síndrome per se es de diagnóstico clínico; éste se establece con: a) Radiografía de tórax AP y lateral, b) ECG, c) cuadro hemático, d) nitrógeno ureico en sangre (BUN), e) creatinina, f) uroanálisis, g) glicemia y h) transaminasas.

\section{INTERVENCIÓN OPORTUNA DEL TRATAMIENTO}

Se basa en tres enfoques: a) Tratar la causa subyacente (cardiopatía congénita, valvulopatías, endocarditis, hipertensión arterial, etc., en caso de ser efectivamente identificado), b) eliminar los factores precipitantes (infecciones, arritmias, tromboembolismo pulmonar, etc.), c) control de la falla cardiaca como bomba teniendo como objetivos mejorar la función de bomba, reducción del trabajo cardiaco en general y control del exceso de agua y sodio.

Los principales medicamentos empleados en el tratamiento convencional de este padecimiento consisten principalmente en: diuréticos, digital, beta-bloqueadores, antiarrítmicos, anticoagulantes e inotrópicos.

La combinación de estos medicamentos es el pilar del tratamiento de la insuficiencia al ser oportuno por muchos años, y desta- cando sobre todo la digoxina, diuréticos y los betabloqueantes; su combinación es la más utilizada, aunque en la actualidad hay nuevas tendencias farmacológicas basadas en detalles fisiopatológicos más precisos que los que éstos comprenden.

En cuanto a la prevención y pronóstico de la insuficiencia cardiaca, no existe una prevención primaria formal, sino más bien intervenciones basadas en diversos niveles de evidencia que permiten disminuir la incidencia de los factores de riesgo asociados, ya que por ser una entidad multifactorial no es claramente predecible el momento o la causa específica de su aparición.

\section{LAS NUEVAS TENDENCIAS FARMACOLÓGICAS EN LA INSUFICIENCIA CARDIACA}

El resultado de numerosos ensayos clínicos ha añadido medicamentos útiles para mejorar los síntomas, retrasar la evolución de la enfermedad y disminuir su mortalidad. ${ }^{32}$ Las estrategias actuales y futuras en el tratamiento de la insuficiencia cardiaca sistólica, diastólica o mixta tienen su fundamento en los avances del conocimiento de la fisiopatología de la propia insuficiencia cardiaca. Cabría mencionar que algunos de los nuevos medicamentos aún se encuentran a nivel experimental o por alguna razón no han podido ser aprobados, pero todos ellos comparten en común un enfoque molecular fisiopatológico.

Aunque son muy diversos los fármacos que se encuentran actualmente en desarrollo, se podrían agrupar de la siguiente manera, según sus principales acciones: bloqueadores de la respuesta neurohormonal, inotrópicos positivos, moduladores del remodelado cardiaco y se debe considerar terapia genética y trasplante, entre otros.

Los diuréticos son imprescindibles para el alivio de los síntomas congestivos, en todos los pacientes con IC sintomática. Existen diuréticos bloqueadores de los receptores de aldosterona; éstos reducen la mortalidad por insuficiencia cardiaca grave (clases III y IV de la NYHA).

Rales (1999) demostró que 30\% reducen la mortalidad con dosis bajas de espironolactona (25-50 mg diarios) en los pacientes con IC, a diferencia de los que consumen IECA. ${ }^{33}$ Los ARA II bloquean el efecto de la angiotensina-II en el receptor específico AT1. Contrario a lo que cabría esperarse, el estudio ELITE II47 no pudo demostrar superioridad de estos fármacos respecto de los IECA en la IC. ${ }^{34} \mathrm{La}$ combinación de un IECA y un bloqueador es la piedra angular del tratamiento de los pacientes con insuficiencia cardiaca por disfunción sistólica del VI. ${ }^{35}$ Todo paciente con disfunción sistólica del VI y síntomas leves o moderados (clases II y III de la NYHA) con tratamiento convencional (diuréticos + IECA) debe ser tratado con bloqueadores, siempre y cuando el paciente conserve estabilidad clínica durante un mes y no existan contraindi- 
caciones como asma, EPOC o bloqueo auriculoventricular. Deben administrarse al inicio dosis bajas, que aumentarán progresivamente cada dos semanas. ${ }^{36}$

Las guías actuales recomiendan la digoxina para los pacientes con síntomas incluso con el uso de diuréticos, IECA y $\beta$-bloqueadores (nivel de evidencia: $\mathrm{B}$ ). Se plantea que tiene una función limitada en los pacientes con ritmo sinusa $1^{15} \mathrm{y}$ que su indicación es en aquellos pacientes con fibrilación auricular con respuesta ventricular rápida. ${ }^{37}$

Los estudios con antagonistas de la endotelina (tezosentán, bosentán) y con antagonistas del TNF- $\alpha$ (etanercept, infliximab) han fracasado. Los primeros producen hepatotoxicidad y no disminuyen la mortalidad, mientras que los segundos están actualmente contraindicados pues incrementan el riesgo de muerte y hospitalización. ${ }^{38,39}$

El levosimendán, un nuevo agente inotrópico con acción inhibidora de la fosfodiesterasa y sensibilizador del calcio, se prescribe en bolos e infusión intravenosa continua en los pacientes con insuficiencia cardiaca avanzada y resistente al tratamiento, ya que mejora la supervivencia a los 180 días. ${ }^{40,41}$

\section{LAS PERSPECTIVAS TERAPÉUTICAS DEL CUIDADO A PROPORCIONAR SON:}

\section{Actividad física}

Revisiones sistemáticas reportan que el ejercicio disminuye la mortalidad y mejora el desempeño de los pacientes con IC, ${ }^{42}$ aunque en nuestros días parece no estar bien reconocido el beneficio en los pacientes, y por otro lado el miedo a establecer un tratamiento que atenta contra uno de los paradigmas es el reto en el tratamiento de la insuficiencia cardiaca. Sin embargo, se requiere del tratamiento quirúrgico y la intervención es en el pre y postoperatorio.

\section{Trasplante cardiaco}

Es una opción terapéutica que mejora considerablemente el pronóstico en los pacientes con IC resistente al tratamiento sin enfermedades concomitantes que contraindiquen el trasplante; también se debe considerar que no hayan procedimientos quirúrgicos de reparación, que la fracción de eyección sea mayor a $20 \%$, la $\mathrm{VO}_{2}$ máxima mayor a $14 \mathrm{~mL} / \mathrm{kg} / \mathrm{min}$ y que en las pruebas de cateterismo cardiaco no exista hipertensión pulmonar irreversible. ${ }^{43}$ Aunque por su parte los trasplantados tienen supervivencia de $80 \%$ al año y de $66 \%$ a los 10 años. ${ }^{44}$

\section{Corazones mecánicos}

Ya existen, en Estados Unidos y Europa, dispositivos destinados a suplir la función del corazón utilizando una bomba de manera ambulatoria, capaz de mantener las demandas del organismo. Estos aparatos se están perfeccionando, y se visualiza que pueden ser temporales en lo que se realiza el trasplante como tratamiento final. El costo es extremadamente alto y la medicación que los acompaña los pone aún lejos de ser la primera opción en el tratamiento de esta enfermedad.

\section{Otros métodos son:}

\section{Terapia celular}

Consiste en el trasplante de mioblastos autólogos o células madre de la médula ósea en zonas infartadas, junto con una operación de revascularización coronaria, misma que ya se está llevando a la práctica de manera muy controlada en algunos centros especializados del mundo, aunque todavía no existen datos de que el tratamiento sea efectivo. Debido al bajo número de casos en los que se ha practicado, aún es insuficiente para establecerlo y todavía se encuentran dilemas que limitan el tratamiento, entre ellos económicos y sobre todo bioéticos.

\section{Grupos de fármacos moduladores neurohormonales}

La IC se acompaña de una marcada activación neurohumoral y se ha demostrado un aumento en los valores plasmáticos de los mediadores (catecolaminas, sistema reninaangiotensina-aldosterona, endotelina 1 , vasopresina) que producen vasoconstricción, retención hidrosalina, edemas y efectos mitogénicos, así como una disminución de los que presentan propiedades vasodilatadoras, natriuréticas y antiproliferativas (péptidos natriuréticos, dopamina, óxido nítrico [NO]), por lo cual se han ido desarrollando varios fármacos, entre ellos: moduladores neurohumorales como péptidos natriuréticos auriculares: nesiritida, vasonatrida, anaritida, mini ANP; inhibidores de vasopeptidasas (IECA+EPN: omapatrilato, fasidotrilo; antagonistas de los receptores de la endotelina ETA): darusentán, sitaxentán; antagonistas del TNF $\alpha$ : pentoxifilina, FR167653, candesartán, $\beta$-bloqueantes); antagonistas de los receptores de la vasopresina (V1A/V2:-conivaptán); V2: tolvaptán [actualmente se encuentra aprobado con resultados prometedores en el estudio EVEREST]; antagonistas de la aldosterona: eplerenona y otros fármacos; antagonistas de los receptores A1 (BF9714); simpaticolíticos: nepicastat, nolomirona; fármacos que mejoran el metabolismo cardiaco: carnitina, etoxomir, hormona del crecimiento, ranolazina, etoxomir, IECAs, $\beta$-bloqueantes, ARAII, espironolactona; antagonistas de los receptores AT1 de la angiotensina II: candesartán, eprosartán, forasartán, irbesartán, olmesartán, ripisartán, tasosartán, telmisartán; nuevos inotrópicos positivos: levosimendán; modulación del remodelado ventricular; in- 
hibidores de metaloproteinasas: hidroxamatos (batimastat, ilomastat, marimastat, prinomastat), $\beta$-bloqueantes, glucocorticoides; también hay que considerar la terapia génica. El objetivo fundamental de la terapia génica es lograr mayor adherencia a los tratamientos sin necesidad de consumir una droga; sólo requerirá atención ocasional aunque se conoce que el costo de estas terapias pudiera ser elevado. ${ }^{45}$

\section{CONCLUSIÓN}

A pesar de los grandes avances realizados en el mundo de la medicina, la IC continúa siendo hoy en día un enorme reto para la práctica clínica, así como una enorme inversión para todos los gobiernos del mundo en materia de salud. Por lo tanto, es indispensable no perder de vista su transición epidemiológica, y con base en ello mantener nuestra atención centrada en comprender cada día mejor los procesos fisiopatológicos que la componen, así como mantener un ímpetu de innovación y conocimiento para poder tratarla de manera eficiente y quizá, con el perfeccionamiento de la farmacología, detener su evolución de manera definitiva.

\section{BIBLIOGRAFÍA}

1. Hunt SA, Abraham WT, Chin MH, Feldman AM et al. ACA/AHA 2005 guideline update for the diagnosis and management of chronic heart failure in the adult-summary article. J Am Coll Cardiol 2005; 46: 1116-43.

2. Ho KK, Pinsky JL, Kannel WB, Levy D. The epidemiology of heart failure: the Framingham study. J Am Coll Cardiol 1993; 22: 6A-13.

3. Rodríguez AF, Banegas BJR, Guallar CP. Epidemiología de la insuficiencia cardiaca. Rev Esp Cardiol 2004; 57: 163-70.

4. Manzano L, Sánchez MD, Rodríguez GP. La insuficiencia cardiaca en la práctica asistencial del internista. Rev Clin Esp 2005; 205 (7): 305-6.

5. European Study Group on Diastolic Heart Failure. How to diagnose diastolic heart failure. Eur Heart J 1998; 19: 990-1003.

6. Opie LH, Commerford PJ, Gersh BJ, Pfeffer MA. Controversies in ventricular remodelling. Lancet 2006; 367: 356-67.

7. Aldama LG, Piñeiro PM, Campo PEP. Insuficiencia cardiaca: Concepto, epidemiología, clasificación, etiología y fisiopatología. Medicine 2005; 9 (35): 2279-90.

8. Cohn JN, Ferrari R, Sharpe NJ, on Behalf of an International Forum on Cardiac Remodeling. Cardiac remodeling - concepts and clinical implications: a consensus paper from an international forum on cardiac remodeling. J Am Coll Cardiol 2000; 35: 569-582.

9. Szokodi I, Kinnunen P, Tavi P, Weckstrom M, Toth M, Ruskoaho H. Evidence for AMP-independent mechanisms mediating the effects of adrenomedullin, a new inotropic peptide. Circulation 1998 97: 1062-1070.

10. Op. cit. 2004; 123 (4): 149-53.

11. Kaluski E, Kobrin I, Zimlichman R, Marmor A et al. RITZ-5: randomized intravenous TeZosentan (an endothelin-A/B antagonist) for the treatment of pulmonary edema: A prospective, multicenter, double blind, placebo-controlled study. J Am Coll Cardiol 2003; 41: 204-10.

12. Pérez VF. Avances en el tratamiento de la insuficiencia cardiaca. Med Clin (Barc) 2004; 123 (4): 149-53.

13. Mann DL. Inflammatory mediators and the failing heart. Past, present, and the foreseable future. Circ Res 2002; 91: 988-98.

14. Goetze JP, Kastrup J, Rehfeld JF. The paradox of increased natriuretic hormones in congestive heart failure patients: Does the endocrine heart also fail in heart failure? Eur Heart J 2003; 24: 1471-2.
15. Doust JA, Pietrzak E, Dobson A, Glasziou P. How well does B-type natriuretic peptide predict death and cardiac events in patients with heart failure: Systematic Review BMJ 2005; 330: 625-33.

16. Braunwald E, Bristow MR. Congestive heart failure: Fifty years of progress. Circulation 2000; 102: 14-23.

17. Herrera-Garza EH, Stetson SJ, Cubillos-Garzon A, VooletichMT, Farmer JA, Torre-Amione G. Tumor necrosis factor-alpha: a mediator of disease progression in the failing human heart. Chest 1999; 115: 1170-1174.

18. Aukrust P, Ueland T, Lien E, Bendtzen K, Muller F, Andreassen AK et al. Cytokine network in congestive heart failure secondary to ischemic or idiopathic dilated cardiomyopathy. Am J Cardiol 1999; 83: 376-382.

19. Bryant D, Becker L, Richardson J, Shelton J, Franco F, Peshock R et al. Cardiac failure in transgenic mice with myocardial expression of tumor necrosis factor-a. Circulation 1998; 97: 1375-1381.

20. Bristow MR. Tumor necrosis factor-a and cardiomyopathy. Circulation 1998; 97: 1340-1341.

21. Mann DL, Spinale FG. Activation of matrix metalloproteinases in the failing human heart: Breaking the tie that binds. Circulation 1998; 98: 1699-1702.

22. Rao VU, Spinale FG. Controlling myocardial matrix remodeling: implications for heart failure. Cardiol Rev 1999; 7: 136-143.

23. Opie LH, Commerford PJ, Gersh BJ, Pfeffer MA. Controversies in ventricular remodelling. Lancet 2006; 367: 356-67.

24. Anderson TJ. Assessment and treatment of endothelial dysfunction in humans. J Am Coll Cardiol 1999; 34: 631-638.

25. Drexler H. Hypertension, heart failure, and endothelial function. Am J Cardiol 1998; 82: 20S-22S.

26. Op cit. Anderson TJ. 1999; 34: 631-638.

27. Bauersachs J, Bouloumié A, Fraccarollo D, Hu K, Busse R, Ertl G. Endothelial dysfunction in chronic myocardial infarction despite increased vascular endothelial nitric oxide synthase and soluble guanylate cyclase expression: Role of enhanced vascular superoxide production. Circulation 1999; 100: 292-298.

28. Negrín JE, Cordiés L, Roselló N, Sánchez J, Negrín JA. Insuficiencia cardiaca crónica. Rev Cubana Med 2001; 40 (3): 195-211.

29. Op cit. European Study Group. 1998; 19: 990-1003.

30. Jessup M, Brozena S. Heart failure. N Engl J Med 2003; 348 (20): 2007-18.

31. Op cit. Hunt SA. Abraham WT, Chin MH, Feldman AM et al. ACA/AHA 2005 guideline update for the diagnosis and management of chronic heart failure in the adult-summary article. J Am Coll Cardiol. 2005; 46: 1116-43.

32. Garrido BJP, Rodríguez FJA, García de LJ, Castro BA. Tratamiento de la insuficiencia cardiaca crónica. Medicine 2005; 9 (35): 2318-26.

33. Pitt B, Zannad F, Remme WJ, Cody R et al. The effect of spironolactone on morbidity and mortality in patients with severe heart failure. N Engl J Med 1999; 341: 709-17.

34. Pitt B, Poole-Wilson P, Segal R, Martínez FA et al. Effects of losartan vs captopril on mortality in patients with symptomatic heart failure: Rationale, design, and baseline characteristics.

35. McMurray JJV, Pfeffer MA. Heart failure. Lancet 2005; 365: 1877-89.

36. Garrido BJP, Rodríguez FJA, García de LJ, Castro BA. Tratamiento de la insuficiencia cardiaca crónica. Medicine 2005; 9 (35): 2318-26.

37. Op cit. Hunt SA. 2005; 46: 1116-43.

38. Op cit. J Am Coll Cardiol 2003; 41: 204-10.

39. Mann DL. Inflammatory mediators and the failing heart. Past, present, and the foreseeable future. Circ Res 2002; 91: 988-98.

40. Muñoz RN. Manejo del paciente con insuficiencia cardiaca aguda e insuficiencia cardiaca refractaria al tratamiento convencional. JANO 2005; 21 (1580): 49-55.

41. Op cit. Garrido Bravo JP, Rodríguez FJA, García de LJ, Castro BA. Tratamiento de la insuficiencia cardiaca crónica. Medicine

42. Mc Kelvie R. Cardiovascular disorders: Heart failure. Interventions. Clinical Evidence. BMJ Publishing Group Limited 2006. URL disponible en: http://www.clinicalevidence.com. Web publication date: 01/04/2006.

43. Op cit. Garrido BJP. 2005; 9 (35): 2318-26.

44. Pérez VF. Avances en el tratamiento de la insuficiencia cardiaca. Med Clin (Barc) 2004; 123 (4): 149-53.

45. Cabrera A. Nuevas estrategias farmacológicas en la insuficiencia cardiaca 14/08/2007. 